Wissen, was wirkt

\title{
Ein Standard für die Berichterstattung von Social Entrepreneurs
}

\section{Social Entrepreneurs oder Sozialunternehmer ge-} hen gesellschaftliche Probleme an und zielen nicht auf die Maximierung des ökonomischen Gewinns. Um die Attraktivität solcher Unternehmungen für Investoren zu steigern, ist eine standardisierte Berichterstattung sinnvoll. Ein solcher Standard muss auf die Besonderheiten von Social Entrepreneurs abgestimmt sein. Von Ann-Kristin Achleitner, Alexander Bassen, Barbara Roder und Lena Lütjens

$V$ eränderungen auf der Angebots- sowie der Nachfrageseite im sozialen Sektor haben einen zunehmenden Professionalisierungsdruck zur Folge, der das Konzept des Social Entrepreneurship begünstigt. Um die tatsächliche Wirksamkeit dieses Ansatzes zu verdeutlichen, fehlte jedoch bisher die Grundlage für eine professionelle Berichterstattung, englisch Reporting, die Geldgebern eine Entscheidungsgrundlage bietet und dem innovativen Konzept des Social Entrepreneurs gerecht wird.

\section{Phänomen Social Entrepreneurship}

Das Phänomen Social Entrepreneurship erfährt in jüngster Zeit verstärkt Aufmerksamkeit. Insbesondere vor dem Hintergrund der Debatte um den Wandel des Sozialstaats und der Ausweitung des Subsidiaritätsgedankens hin zu mehr Eigenverantwortlichkeit kommt Social Entrepreneurs eine zunehmend wichtige Bedeutung zu. Dabei sehen sich Social Entrepreneurs zunehmend auch mit der Messung ihres Erfolgs, der Darstellung ihres Risikos sowie einer professionellen Dokumentation ihrer Arbeit konfrontiert. Es existiert jedoch bisher kein einheitlicher Reporting Standard, der an die Bedürfnisse von Social Entrepreneurs angepasst ist und in dem Informationen systematisch und strukturiert erfasst werden.

Eine an Indikatoren orientierte, standardisierte Berichterstattung von Social Entrepreneurs würde verschiedenen Zielsetzungen dienen. Sie ermöglicht eine Vergleichbarkeit verschiedener Organisationen innerhalb eines Themenbereichs hinsichtlich ihrer Effektivität sowie zwischen allen Organisationen hinsichtlich ihrer Effizienz. Darüber hinaus könnten die Informationen über die Performance der Organisationen durch Analysten bewertet werden. Dies wiederum ermöglicht Investoren eine Allokationsentscheidung nicht nur hinsichtlich ihrer Präferenz für ein bestimmtes gesellschaftliches Thema, vielmehr könnten dadurch auch persönliche Risiko- und Return-Profile be- rücksichtigt werden. Des Weiteren wären Social Entrepreneurs in der Lage, ein solches professionelles Wirkungsmonitoring auch für interne Managemententscheidungen zu nutzen. Eine standardisierte Messung und der Ausweis des sozialen Erfolgs könnten so zu einem geringeren Risiko und größerem Vertrauen aufseiten der Geldgeber und somit letztlich zu einer effizienteren Kapitalallokation im Dritten Sektor führen.

\section{Globalfunktion des Reporting}

Werden Informationsbedürfnisse von Investoren durch Unternehmen nicht erfüllt, sind die aufgrund asymmetrischer Informationsverteilung entstandenen hohen Kosten der Informationsbeschaffung oft ausschlaggebend dafür, dass eine Investition nicht erfolgt (Achleitner/Behr 2009). Um vor Vermögensverlusten durch Fehlentscheidungen bewahrt zu werden, benötigen Investoren eine aussagefähige Berichterstattung über Unternehmen (Moxter 2003). Je transparenter und vergleichbarer diese Informationen sind, desto besser lassen sich zukünftige Renditen schätzen und Risiken einer Investition minimieren. Umso attraktiver wird damit ein Unternehmen aus Sicht des Investors (AICPA 1997; Ernst 2002). Diese Erleichterung der Kapitalaufnahme führt dann auch zu einer Senkung der Kosten der Kapitalbeschaffung und einer effizienteren Kapitalallokation (Ernst 2002; Wagenhofer 2002).

Reporting kann im Deutschen am treffendsten mit externer Unternehmensberichterstattung übersetzt werden. Ein Reporting umfasst alle Informationen eines Unternehmens, die die Reportingadressaten bei der Beurteilung der Aktivitäten des Berichtenden maßgeblich unterstützen. Als Reportingadressaten mit originärem Informationsbedarf können im Social Entrepreneurship öffentliche Einrichtungen, private Investoren, ehrenamtliche Mitarbeiter sowie Kunden ermittelt werden. Die interessierte Öffentlichkeit sowie Finanzintermediäre sind indirekte Adressaten mit derivativem Informationsbedarf.

\section{Status quo der Erfolgsmessung}

Traditionelles Reporting, in dem fast ausschließlich finanzielle Kennzahlen erhoben werden, ist bei Social Entrepreneurs nicht ausreichend. Zum einen hängt die Möglichkeit, überhaupt Einkommen zu generieren, davon ab, welches gesellschaftliche Problem der Social Entrepreneur bekämpft. Zum anderen ist für Social Entrepreneurs die monetäre Zielerreichung sekundär, sie streben vielmehr nach gesellschaftlichem Wandel. 
Die Messung dieses sozialen Erfolgs ist jedoch mit zahlreichen methodischen Schwierigkeiten verbunden. In diesem Zusammenhang sind vor allem die komplexe Operationalisierung der Wirkungsziele aufgrund ihrer qualitativen Natur, die problematische Erfassung und Messung von Kausalitätszusammenhängen sowie eine starke Subjektivität der Wirkung anzuführen. Aufgrund dieser vielschichtigen Herausforderungen lässt sich der Status quo der Evaluierungspraxis als stark fragmentiert und uneinheitlich bezeichnen. Es hat sich bis dato keine standardisierte und objektive Kennzahl zur Messung sozialer Wertschöpfung durchgesetzt. Zwar wurden bereits Konzepte zur Messung nicht-finanzieller Ziele entwickelt, die meisten davon jedoch für einen spezifischen Anlass. Defizite bestehen vor allem in der Entwicklung eines wissenschaftlich fundierten Reportingkonzepts und eines darauf aufbauenden Reportingleitfadens für Social Entrepreneurs. Konsequenz dieser Schwierigkeiten der Erfolgsmessung ist unter anderem, dass Social Entrepreneurs unabhängig von ihrer Leistung Finanzierungsprobleme haben, was als Indiz für einen ineffizienten Markt gesehen werden kann.

Wesentliche Informationen, die es dem Berichtspublikum ermöglichen, einen Social Entrepreneur einzuschätzen, betreffen hauptsächlich seinen Erfolg. Erfolgsbezogene Informationen umfassen den effektiven und effizienten Ressourceneinsatz zur Erreichung eines bestimmten Ziels innerhalb eines definierten Zeitraums (Twersky/Blair 2002;). Der Erfolg einer Organisation wird somit gemessen am Grad der spezifischen Zielrealisation und gibt darüber Aufschluss, in welchem Maß das angestrebte Ziel erreicht wurde. Zu beachten ist in diesem Zusammenhang eine klare Sachzieldominanz bei Social Entrepreneurs gegenüber der Formalzieldominanz bei privatwirtschaftlichen Unternehmen (Eichhorn 2001). Für diese soziale Zielsetzung von Social Entrepreneurs existiert bis dato keine monetäre Zielgröße. Sie lässt sich auch nicht objektiv ermitteln, da die Leistungen von Social Entrepreneurs nicht durch Preise auf freien Märkten bewertet werden. Damit fehlt Social Entrepreneurs das zentrale Rechnungsziel privatwirtschaftlicher Unternehmen. Der Informationsbedarf über den Organisationserfolg, das heißt die Sachzielerreichung von Social Entrepreneurs, muss daher durch ein für diesen Unternehmertyp spezifisches Reporting gedeckt werden (Küpper 2007).

Neben dem Erfolg des Social Entrepreneurs müssen auch diejenigen Faktoren in einem Reporting berücksichtigt werden, die Einfluss auf die Zielerreichung haben. Dies ist zum einen das Risiko (Budäus/Hilgers 2009). Das Eingehen von Risiken ist ein Kernelement unternehmerischen Handelns und trifft auch auf Social Entrepreneurs zu. Ein funktionsfähiges und kontinuierlich betriebenes Risikomanagement birgt mehrere Vorteile. Die Darstellung der mit dem eigenen Konzept verbundenen Risiken ist für den Social Entrepreneur selbst ein wichtiger Bewusstseinsschritt. Auch werden das Vertrauen und die Planungssicherheit von Mitarbeitern und Unterstützern gestärkt (Herman 2005). Für Investoren schließlich stellt das Reporting von Risiken eine Entscheidungsgrundlage dar, mit dem sie ihre Investitionen ent- sprechend ihrem Rendite-Risiko-Profil optimieren können (Achleitner et al. 2009).

\section{Aufbau des Reporting Standards}

Ein zweiter Einflussfaktor auf den Erfolg von Social Entrepreneurs, der in einem Reporting angeführt werden sollte, ist die Leistungsfähigkeit der Organisation, die sogenannte Organizational Capacity. Im Gegensatz zum Erfolg, das heißt der Frage, was eine Organisation erreicht hat, beschäftigt sich die Organizational Capacity damit, wie und durch wen Erfolg erreicht wird (Connolly/Lukas 2004;). Sie kann definiert werden als „the organisation's ability to survive, to successfully apply its skills and resources in order to pursue its goals and satisfy its stakeholders' expectations" (Horton et al. 2003). Organizational Capacity ist abhängig von Alter und Größe einer Organisation (Tobelem 1992).

Der Ansatz, den Aufbau leistungsfähiger Organisationen voranzutreiben, sogenanntes Organization Building, kommt aus der Venture-Capital-Literatur und wurde im sozialen Sektor vor allem von Venture-Philanthropy-Funds und einigen Stiftungen übernommen (Letts/Ryan/Grossman 1997). Im Gegensatz zu bisher vorrangig geförderten operativen Programmen nimmt die Bedeutung des zur Umsetzung dieser Programme notwendigen Organisationsaufbaus zu (Letts/Ryan/Grossman 1997). Dem liegt die Annahme zugrunde, dass eine Organisation nachhaltig erfolgreicher und innovativer sein kann, wenn sie leistungsfähiger ist.

Um den genannten Anforderungen Rechnungen zu tragen, müssen die folgenden Parameter in ein Reporting für Social Entrepreneurs einbezogen werden:

Das Konzept des Social Entrepreneurs ist stark personenzentriert und geht davon aus, dass ein bedeutender Teil des von ihm angestoßenen sozialen Wandels auf seine unternehmerischen Fähigkeiten zurückzuführen ist. Abgeleitet aus Studien im Venture-Capital-Bereich beinhaltet das Reportingmodell deshalb Informationen über die Persönlichkeit des Social Entrepreneurs, seine Gründungs- und Führungserfahrung sowie seine Kompetenzen (Knight 1994).

\section{Organizational Capacity}

Maßgeblich für eine Investitionsentscheidung ist neben der Person des Social Entrepreneurs auch die Leistungsfähigkeit seiner Organisation. Die Erfassung der Organizational Capacity im Reporting für Social Entrepreneurs basiert auf dem St. Galler Management Modell sowie dem Konzept des integrierten Managements (Ulrich/Krieg 1974; Bleicher 2001; Schwaninger 2006).

Kern dieses Modells sind die drei Planungsebenen des integrierten Managements: Normativ, strategisch und operativ (Bleicher 2001; Schwaninger 2006). Das normative Management beschäftigt sich mit den generellen Zielen einer Organisation, die darauf ausgerichtet sind, die Lebens- und Entwicklungsfä- $\rightarrow$ 
higkeit sicherzustellen und richtet sich auf die Nutzenstiftung für Stakeholder (Bleicher 2001). Übertragen auf ein Reporting im Social Entrepreneurship umfasst die normative Ebene daher die Beschreibung des sozialen Problems, das bekämpft wird, sein Ausmaß und die Darstellung existierender konventioneller sowie alternativer Lösungsansätze, um das Umfeld und damit die Notwendigkeit einer Intervention durch den Social Entrepreneur zu begründen. Darüber hinaus enthält sie seine Vision und seine langfristigen Ziele.

Da der normative Rahmen verschiedene strategische Möglichkeiten bietet, spezifiziert die strategische Ebene die Art und Weise, wie eine Organisation ihre Vision erreichen möchte (Schwaninger 2001; Bleicher 2001). Auf der strategischen Ebene eines Reporting im Social Entrepreneurship wird der besondere Lösungsansatz des Social Entrepreneurs, seine Theory of Change dargestellt (Twersky/Blair 2002). Die verschiedenen Zielgruppen sowie die Funktionsweise des Geschäftsmodells des Social Entrepreneurs, die Organisationsmerkmale Innovation, Einkommensgenerierung, Replizierbarkeit, Position des Unternehmens im Lebenszyklusmodel sowie deren Ausprägung spielen hier eine entscheidende Rolle.

Die Ebene des operativen Managements schließlich beschäftigt sich mit der Implementierung des Lösungsansatzes. Sie enthält daher alle Informationen über leistungs-, finanz- und personalwirtschaftliche Prozesse und gibt einen Überblick über sämtliche Inputfaktoren, die der Social Entrepreneur für sein Streben nach gesellschaftlichem Wandel einsetzt (Bleicher 2001).

Um die gesellschaftliche Wirkung von Social Entrepreneurs zu erfassen, wird auf das Modell der Impact Value Chain zurückgegriffen (Clark et al. 2004). Die Planungsebenen des integrierten Managements, normativ, strategisch und operativ, werden in der Reportingkonzeption logisch verknüpft mit den Wirkungs- und Leistungsebenen Outcome, Impact und Output. Kernelemente der normativen Ebene sind die unternehmerische Vision und das Ziel des Social Entrepreneurs, einen bestimmten gesellschaftlichen Missstand zu bekämpfen. In der Art der Zielsetzung liegt somit der Hauptunterschied zwischen einem Social Entrepreneur und dem klassischen Business Entrepreneur begründet. Erfolg, definiert als Grad der Zielerreichung, kann auf dieser Ebene gemessen werden durch den Indikator Outcome, der intendierten zielbezogenen generellen Wirkung.

Der Beitrag des jeweiligen Social Entrepreneurs zu dieser positiven gesellschaftlichen Veränderung ist determiniert durch die spezifische strategische Aufstellung, der Theory of Change, seiner Organisation. Dieser Erfolg kann durch den Indikator Impact gemessen werden, hierbei handelt es sich um ,the difference between the outcome for a sample exposed to an enterprise's activities and the outcome that would have occurred without the venture or organization" (Clark et al. 2004). Die Operationalisierung und Erhebung von Impact stellt eine besondere Herausforderung dar, die in Abhängigkeit vom Tätigkeitsbereich des Social Entrepreneurs stark variiert. Im Rahmen einer ausführlichen Analyse der eigenen Wirkungskette sowie der Einbindung der wichtigsten Stakeholder bei der Festelegung der Messindikatoren kann versucht werden, sich einer ImpactBerechnung zu nähern. In Anlehnung an die Methode des Social Return on Investment sollte eine ausführliche Impactmessung sowohl positive wie auch unintendierte negative Wirkungen mit einbeziehen.

Der Erfolg auf normativer und strategischer Ebene kann durch Outcome und Impact absolut und durch ein Effektivitätsziel relativ gemessen werden. Auf operativer Ebene ist der Indikator Output das absolute Maß der Performance und das relative Verhältnis von Output zu Input ermöglicht Aussagen über die Effizienz.

Die Darstellung der mit dem eigenen Konzept verbundenen Risiken ist ein wichtiger Bewusstseinsschritt für den Social Entrepreneur selbst und außerdem eine wichtige Entscheidungsgrundlage für mögliche Investoren. Die Erreichung der gesetzten Ziele ist immer einem gewissen Risiko ausgesetzt. Die Charakteristika, die die Erfolgsmessung nicht-finanzieller Ziele beeinflussen, wirken deshalb ebenso auf das Risiko. Deshalb werden auf jeder Ebene spezifische Risikoparameter definiert.

Die Umsetzung der theoretischen Reportingkonzeption in einen Reporting Standard für Social Entrepreneurs ergibt folgenden Aufbau:

I Das gesellschaftliche Problem

In einem ersten einleitenden Block wird das gesellschaftliche Problem, das bekämpft wird, beschrieben. Es wird das Tätigkeitsfeld bestimmt, das Ausmaß des gesellschaftlichen Misstandes angeführt sowie bisherige Lösungsansätze dargestellt.

I Die Theory of Change

In einem zweiten Teil legt der Social Entrepreneur dann seine Theory of Change dar. Er beschreibt detailliert seinen Lösungsansatz, seine Kernzielgruppe sowie weitere Stakeholder und erläutert Details zum Geschäftsmodell sowie damit verbundene Risiken.

I Die Organisation

Der dritte Abschnitt beinhaltet Informationen über die Organisation des Social Entrepreneurs. Angegeben werden hier ein Organisationsprofil, ökonomische Leistungsindikatoren, Revenue-Modelle sowie Organisationsziele und Milestones für die nächsten zwölf Monate.

- Der Social Entrepreneur

An vierter Stelle wird der Social Entrepreneur vorgestellt, seine Erfahrung, Kompetenz sowie Motivation und Ideenfindung.

I Wirkungsmessung

Zentraler Bereich des Reportings ist die Wirkungsmessung, die am Ende des Berichts dargestellt wird. Angeführt werden an dieser Stelle die Bemessung und Darstellung der Outputs, Outcomes sowie des erzielten Impacts. Dies erfolgt beim Output quantitativ, unterteilt in personen-, institutionsund aktivitätsbezogenen Output. Outcome und Impact werden hauptsächlich qualitativ erfasst, es besteht jedoch die 
Option, wenn möglich und angebracht, diese Parameter auch zu quantifizieren.

\section{Erfahrungen aus der praktischen Anwendung}

Die Konzeption und erste Anwendung des Reporting Standards für Social Entrepreneurs erstreckte sich über einen Zeitraum von zwei Jahren und durchlief die folgenden Phasen:

I Theoretische Entwicklung der Reporting Guidelines für Social Entrepreneurs.

- Erster Pretest: persönliche Befragung von sieben Social Entrepreneurs zur Ermittlung praxisorientierter Notwendigkeiten eines Reportings in Form von leitfadengestützten Interviews.

- Überarbeitung und Einarbeitung der Ergebnisse des Pretests in die Reporting Guidelines. Plausibilisierung durch Interviews mit aktuellen sowie potenziellen Investoren im Social Entrepreneurship.

- Einbezug und Kooperation mit möglichst vielen Akteuren aus dem Bereich Social Entrepreneurship in Deutschland.

- Zweiter Pretest: Erstellung eines Reportings auf der Grundlage der entwickelten Guidelines für alle deutschen und schweizerischen Ashoka-Fellows und Schwab Social Entrepreneurs. Kontinuierliche Adaption des theoretischen Modells.

- Überarbeitung und Einarbeitung der Ergebnisse des Pretests in die Reporting Guidelines.

【 Programmierung eines clientbasierten Tools für alle Social Entrepreneurs sowie kompatible Datenbanken bei Investoren.

- Launch des neuen Reporting Standards und Veröffentlichung der Reporting-Guidelines als Open Source online.

- Erste Gespräche mit einer Wirtschaftsprüfungsgesellschaft hinsichtlich einer Testierbarkeit des jahresabschlussähnlichen Reportings.

Im Rahmen des Kooperationsprojektes wurden für alle 27 Ashoka Social Entrepreneurs in Deutschland und der Schweiz auf der Grundlage von Interviews Reports nach dem hier vorgestellten Konzept erstellt. Als sozialer Investor sucht und fördert Ashoka seit 1980 in fast 70 Ländern Social Entrepreneurs. Diese sogenannten Fellows oder Stipendiaten erhalten von Ashoka finanzielle Unterstützung, Beratung und Anschluss an Netzwerke im sozialen Sektor sowie in Wirtschaft und Wissenschaft, damit sie ihre Projekte verbreiten können. Mit der Anwendung der von der Technischen Universität München und der Universität Hamburg entwickelten neuen Methodik der Berichterstattung und Wirkungsmessung für alle deutschen und schweizerischen Ashoka Fellows möchte die Organisation einen aktiven Beitrag zur Professionalisierung von Sozialunternehmertum leisten.

In diesem Zusammenhang erfüllt der neue Reportingstandard für die Social Entrepreneurs gleich mehrere Zielsetzungen:

I Internes Monitoring: Hilfsmittel für die Eigenevaluation und Weiterentwicklung der Initiative.

- Außendarstellung: Grundlage der eigenen Darstellung der Wirkungsweise und der Erfolge.
I Investorensuche: Darstellung des sozialen Impacts eines Investments oder einer Zuwendung sowie Einschätzung benötigter Mittel und der Skalierbarkeit der Wirkung.

I Rechenschaftslegung: Grundlage für einheitliches Reporting an staatliche Institutionen, private Investoren oder an einer Replizierung interessierte Menschen.

Im Rahmen der Interviews mit den Social Entrepreneurs konnte der Bedarf eines Reporting Standards gezielt für diese Gruppe ausnahmslos bestätigt werden. In Bezug auf die Schwerpunktsetzung seiner Anwendung variierten die Erwartungen der Fellows jedoch in Abhängigkeit vom jeweiligen Entwicklungsstand der Organisation hinsichtlich der Gewichtung der vorrangigen Zielsetzung.

\section{Schlussfolgerungen und Ausblick}

Die Messung positiven gesellschaftlichen Wandels ist zu einem großen Teil immer auch subjektiv beeinflusst von den Wertvorstellungen der beteiligten Personen. Es ist außerdem unwahrscheinlich, dass es in nächster Zeit gelingen kann, rein eindimensionale Kausalitätszusammenhänge für erzielte soziale Wirkungen festzustellen. Aber selbst wenn es unmöglich sein sollte, für den Erfolg von Social Entrepreneurs eine sogenannte Bottom Line zu ermitteln, die mit der Eindeutigkeit ökonomischen Profits vergleichbar wäre, so ermöglicht eine transparente Erfolgsmessung dennoch intertemporale Vergleiche und bessere Analysen innerhalb spezifischer Themenfelder. In diesem Zusammenhang erfasst ein standardisiertes Reporting auch wichtige Daten für wissenschaftliche Längsschnittstudien, beispielsweise im Bereich Finanzierung. Weitere Untersuchungen sollten sich darüber hinaus vor allem auf die Untersuchung von Ursache-Wirkungs-Beziehungen sowie die Attributionsproblematik konzentrieren. In Bezug auf seine theoretische Weiterentwicklung ist das Reporting so konzipiert, dass es als Grundlage für weitere Analysen wie eine Social-Return-on-Investment-Berechnung anschlussfähig ist und sich auch in unternehmensinterne Monitoringsysteme integrieren lässt.

Das vorgestellte Konzept eines Reportingstandards für Social Entrepreneurs erhebt keinen universellen Gültigkeitsanspruch. Vielmehr soll es die Etablierung einer professionellen Berichterstattung und Wirkungsmessung im Social Entrepreneurship fördern, um so zu einer effizienteren Ressourcenallokation im sozialen Sektor beizutragen. Diese Bemühung wird von zahlreichen Investoren und Intermediären im Bereich Social Entrepreneurship unterstützt, die sich der Initiative bereits angeschlossen haben und die Einführung und Weiterentwicklung des Reporting Standards vorantreiben.

\section{Literatur}

Achleitner, A.-K. / Behr, G.: International Accounting Standards: Ein Lehrbuch zur internationalen Rechnungslegung. München 2009.

AICPA: Improving Business Reporting - A Customer Focus. 1997.

Baetge, J. / Kirsch, H.-J. / Thiele, S.: Bilanzen. Düsseldorf 2007.

Bernstein, P.L.: Wider die Götter: die Geschichte der modernen Risikogesellschaft. Hamburg 2004. 
Bleicher, K.: Das Konzept integriertes Management: Visionen, Missionen, Programme. Frankfurt/Main 2001.

Brinckerhoff, P.-C.: Social Entrepreneurship: The Art of Mission-Based Venture Development. New York u.a. 2000.

Budäus, D. / Hilgers, D.: Öffentliches Risikomanagement - zukünftige Herausforderungen an Staat und Verwaltung. In: Scholz, F. / Schuler, A. / Schwintowski, H.-P. (Hrsg.): Risikomanagement der Öffentlichen Hand. Heidelberg 2009. S. 17-77.

Cantillon, R.: Essay on the nature of commerce in general. New York 1931

Clark, C. / Rosenzweig, W. / Long, D. / Olsen, S.: Double Bottom Line Project Report: Assessing Social Impact in Double Line Ventures. 2004.

Cohen, J.M.: Building Sustainable Public Sector Managerial, Professional, and Technical Capacity: A Framework für Analysis and Intervention. Harvard 1993.

Connolly, P. / Lukas, C.: Strengthening nonprofit performance: A funder's guide to capacity building. St. Paul 2004

Eichhorn, P.: Konstitutive Merkmale von Non-Profit-Organisationen. In: Witt, D. / Eckstaller, C. / Faller, P. (Hrsg.): Non-Profit-Management im Aufwind? Wiesbaden 2001. S. 45-52.

Emerson, J.: Understanding Risk: The Social Entrepreneur, and Risk Management. In: Dees, J.G. / Emerson, J. / Economy, P. (Hrsg.): Enterprising nonprofits: a toolkit for social entrepreneurs. New York 2001. S. 125-160.

Ernst, E.: Internationale Harmonisierung der Rechnungslegung und ihre Fortentwicklung - Anforderungen an börsennotierte Großkonzerne in Deutschland. In: zfbf Schmalenbachs Zeitschrift für Betriebswirtschaftliche Forschung 54/2002. S. 181-190.

Financial Accounting Standards Board: Statement of Financial Accounting Concepts No. 2. Norwalk 1980.

Haller, A.: Wesentliche Ziele und Merkmale US-amerikanischer Rechnungslegung. In: Ballwieser, W. (Hrsg.): US-amerikanische Rechnungslegung. Stuttgart 1995. S. 1-26.

Hebert, R.F. / Link, A.N.: In Search of the Meaning of Entrepreneurship. In: Small Business Economics 1, 1/1989. S. 39-49.

Herman, M.L.: Risk Management. In: Herman, R.D. (Hrsg.): The Jossey-Bass Handbook of Nonprofit Leadership and Management. San Francisco 2005. S. 560-584.

Honadle, B.W.: Defining and Doing Capacity Building: Perspectives and Experiences. In: Honadle, B.W. / Howitt, A.M. (Hrsg.): Perspectives on Management Capacity Building. New York 1986. S. 9-23.

Horton, D. / Alexaki, A. / Bennett-Lartey, S. / Brice, K.N. / Campilan, D. / Carden, F. / De Souza Silva, J. / Thanh Duong, L. / Khadar, I. / Maestrey Boza, A. / Muniruzzaman, I.K. / Perez, J. / Somarriba Chang, M. / Vernooy, R. / Watts, J.: Evaluating Capacity Development: Experiences from Research and Development around the World. The Hague u.a. 2003.

Institut der Deutschen Wirtschaftsprüfer: Entwurf für Grundsätze ordnungsmäßiger Prüfung oder prüferischer Durchsicht von Berichten im Bereich der Nachhaltigkeit. 2005.

John, R.: Beyond The Cheque: How Venture Philanthropists Add Value. Oxford 2007.

Knight, F.: Risk, Uncertainty, and Profit. Boston 1921.

Knight, R.M.: Criteria Used by Venture Capitalists: A Cross Cultural Analysis. In: International Small Business Journal 13, 1/1994. S. 26-37.

KPMG: Rechnungslegung nach US-amerikanischen Grundsätzen: Grundlagen der US-GAAP und SEC-Vorschriften. Düsseldorf 2003

Kuhlewind, A.-M.: Grundlagen einer Bilanzrechtstheorie in den USA. München 1997.

Küpper, H.-U.: Neue Entwicklungen im Hochschulcontrolling. In: Controlling \& Management, Sonderheft 3/2007. S. 82-90.

Küting, K. / Reuter, M.: Bilanzierung im Spannungsfeld unterschiedlicher Adressaten. In: DSWR Datenverarbeitung, Steuer, Wirtschaft, Recht 33, 9/2004. S. 230-233.

Letts, C. / Ryan, W. / Grossman, A.: Virtuous Capital: What Foundations Can Learn from Venture Capitalists. In: Harvard Business Review 75, 2/1997. S. 36-44.

McPhee, P. / Bare, J.: Introduction. In: DeVita, C.J. / Fleming, C. (Hrsg.): Building Capacity in Nonprofit Organizations. Washington, D.C. 2001

Meehan III, W.F. / Kilmer, D. / O'Flanagan, M.: Investing in Society - Why we need a more efficient social capital market and how we can get there.
In: Stanford Social Innovation Review, Spring 2004. S. 33-43.

Mikus, B.: Risiken und Risikomanagement - ein Überblick. In: Götze, U. / Henselmann, K. / Mikus, B. (Hrsg.): Risikomanagement. Heidelberg 2001. S. 3-28.

Moock, J.L.: Rockefeller Foundation: How We Invest in Capacity Building. New York 2004

Mort, G.S. / Weerawardena, J. / Carnegie, K.: Social entrepreneurship: Towards conceptualisation. In: International Journal of Nonprofit \& Voluntary Sector Marketing 8, 1/2003. S. 76 - 88.

Moxter, A.: Fundamentalgrundsätze ordnungsgemäßer Rechenschaft. In: Baetge, J. / Moxter, A. / Schneider, D. (Hrsg.): Bilanzfragen. Düsseldorf 1976. S. 89-99.

Moxter, A.: Grundsätze ordnungsmäßiger Rechnungslegung. Düsseldorf 2003.

Otte, R.: Konzernabschlüsse im öffentlichen Bereich: Notwendigkeit und Zwecke konsolidierter Jahresabschlüsse von Gebietskörperschaften dargestellt am Beispiel der Bundesverwaltung der Bundesrepublik Deutschland. Frankfurt a. M. u.a. 1990.

Pietsch, G.: Die Informationsökonomik in der Controllingforschung. In: Scherm, E. / Pietsch, G. (Hrsg.): Controlling: Theorien und Konzeptionen. München 2004. S. 143-165.

Sandberg, B.: Rechnungslegung von Stiftungen - Überlegungen zur Anwendung handelsrechtlicher Vorschriften. In: ZHR Zeitschrift fü das gesamte Handelsrecht und Wirtschaftsrecht 164/2000. S. 155-175.

Schwaninger, M.: Intelligent organizations: an integrative framework. In: Systems Research \& Behavioral Science 18, 2/2001. S. 137-158

Schwaninger, M.: Intelligent Organizations: Powerful Models for Systemic Management. Heidelberg 2006.

Tobelem, A.: Institutional Capacity Analysis and Development System (ICADS). Operation Manual. Washington, D.C. 1992.

Twersky, F. / Blair, J.: Performance Information That Really Performs In: Dees, J.G. / Emerson, J. / Economy, P. (Hrsg.): Strategic Tools for Social Entrepreneurs: Enhancing the Performance of Your Enterprising Nonprofit. New York 2002. S. 161-188.

Ulrich, H. / Krieg, W.: St. Galler Management-Modell. Bern 1974.

Wagenhofer, A.: Die Rolle der Standards des IASB in der Internationalisierung der Rechnungslegung. In: BFuP Betriebswirtschaftliche Forschung und Praxis 54, 3/2002. S. 230-245.

\section{AUTOREN + KONTAKT}

Dr. Dr. Ann-Kristin Achleitner ist Leiterin und Dipl.-Kffr. Barbara Roder ist wissenschaftliche Mitarbeiterin am KfW-Stiftungslehrstuhl für Entrepreneurial Finance der TUM Business School an der Technischen Universität München.

KfW-Stiftungslehrstuhl für Entrepreneurial Finance, TUM Business School, Technische Universität München, Arcisstr. 21, 80333 München Tel.: +49 89 28925-181, Fax: +49 89 28925-188, E-Mail: ann.kristin-achleitner@wi.tum.de. barbara.roder@wi.tum.de

Dr. Alexander Bassen ist Professor für Allgemeine Betriebswirtschaftslehre mit Schwerpunkt Finanzierung und Investition an der Universität Hamburg.

Lehrstuhl für Allgemeine Betriebswirtschaftslehre, Fakultät für Wirtschafts- und Sozialwissenschaften, Universität Hamburg, 20146 Hamburg. Tel.: +4940 428387549, Fax: +49 40428386272 , E-Mail: alexander.bassen@wiso.uni-hamburg.de M.A.-Soz. Lena Lütjens ist Mitarbeiterin bei Ashoka Deutschland.

Ashoka Deutschland gGmbH, Taunustor 2, 60311 Frankfurt. Tel.: +496971625588, E-Mail: Iluetjens@ashoka.org
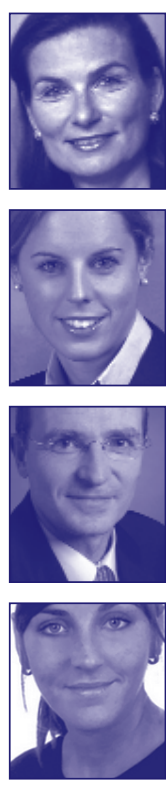
(c) 20I0 Authors; licensee IÖW and oekom verlag. This is an article distributed under the terms of the Creative Commons Attribution Non-Commercial No Derivates License (http://creativecommons.org/licenses/by-nc-nd/3.o/), which permits unrestricted use, distribution, and reproduction in any medium, provided the original work is properly cited. 